3 days) compared with controls (median 2 days; IQR 1-5 days) $(p=0.04)$ [5], suggesting a more rapid onset of symptoms in children at greater risk of hospital presentation for pneumonia. We now show that the same cases were less likely to have visited a GP for other illnesses in the previous year. The lack of association between these two variables $(p=0.8)$ suggests the two findings are unrelated.

We therefore hypothesise that a child presenting to general practice promptly after illness onset, with a history of infrequent consulting, may be at increased risk for pneumonia or empyema. This is consistent with common knowledge regarding any illness that needs prompt and decisive action by the GP. However, our findings cannot inform GP decision making because they may be influenced by response bias. Larger, prospective studies are needed to test our hypothesis.

\section{Joanna C. Crocker*, Colin V.E. Powell ${ }^{\#}$, Kerenza Hood ${ }^{*}$,} Christopher C. Butler* and Meirion R. Evans*

*Institute of Primary Care and Public Health, School of Medicine, Cardiff University, Cardiff, "Dept of Child Health, Institute of Molecular and Experimental Medicine, School of Medicine, Cardiff University, Cardiff, and ${ }^{\top}$ South East Wales Trials Unit, School of Medicine, Cardiff University, Cardiff, UK.

Correspondence: J.C. Crocker, Louis Dundas Centre, UCL Institute of Child Health, 30 Guilford Street, London, WC1N 1EH, UK. E-mail: joanna.crocker@ucl.ac.uk

Support Statement: This study was part of a PhD Studentship funded by the Medical Research Council (grant number G0600288). Additional funding was provided by the British Society for Antimicrobial Chemotherapy (grant number GA854), Royal College of General Practitioners (grant number SFB-2009-01) and the National Institute for Social Care and Health Research (NISCHR), Welsh Government. The study was supported by the South East Wales Trials Unit and the Wales School for Primary Care Research, both funded by NISCHR.

Statement of Interest: None declared.

Acknowledgements: We are most grateful to all the clinicians who recruited patients for the study; lead collaborators: Anjum Gandhi and Ian Hodges (Royal Glamorgan Hospital, Pontyclun, UK), David Deekollu (Prince Charles Hospital, Merthyr Tydfil, UK), Kate Creese and Nirupa D'Souza (Princess of Wales Hospital, Bridgend, UK), Marcus Pierrepoint and Yvette Cloete (Nevill Hall Hospital, Abergavenny, UK), Michelle Barber (Royal Gwent Hospital, Newport, UK and Rachel Evans (Morriston Hospital, Swansea, UK); Hayley Prout (Cardiff University, Cardiff, UK) for recruiting general practices; Ann Russell, Anwen Howells and Marie Williams (NISCHR Clinical Research Centre, Cardiff, UK) for collecting medical data; all participating general practices; and, above all, the carers and children who took part in the study.

\section{REFERENCES}

1 Heiskanen-Kosma T, Korppi M, Jokinen C, et al. Risk factors for community-acquired pneumonia in children: a population-based case-control study. Scand J Infect Dis 1997; 29: 281-285.

2 Teepe J, Grigoryan L, Verheij TJ. Determinants of communityacquired pneumonia in children and young adults in primary care. Eur Respir J 2010; 35: 1113-1117.

3 Shah N, Ramankutty V, Premila PG, et al. Risk factors for severe pneumonia in children in south Kerala: a hospital-based casecontrol study. J Trop Pediatr 1994; 40: 201-206.

4 Fatmi Z, White F. A comparison of "cough and cold" and pneumonia: risk factors for pneumonia in children under 5 years revisited. Int J Infect Dis 2002; 6: 294-301.

5 Crocker JC, Powell CV, Evans MR, et al. Paediatric pneumonia or empyema and prior antibiotic use in primary care: a case-control study. J Antimicrob Chemother 2012; 67: 478-487.

6 Austin H, Hill HA, Flanders WD, et al. Limitations in the application of case-control methodology. Epidemiol Rev 1994; 16: 65-76.

\title{
Asymmetric dimethylarginine and asthma: results from the Childhood Asthma Prevention Study
}

\section{To the Editor:}

Asymmetric dimethylarginine (ADMA) is a naturally occurring analogue of L-arginine and functions as an endogenous inhibitor of nitric oxide synthase (NOS). ADMA is an established risk factor for cardiovascular disease and contributes to chronic endothelial dysfunction [1]. Recently, it has been proposed that ADMA is also a mediator of allergic airways disease, with animal studies indicating a possible role in the development of airway hyperresponsiveness, lung inflammation and fibrosis. Exogenous administration of ADMA has been shown to augment airway responsiveness to methacholine in murine models of asthma and increased ADMA levels were observed in lung homogenates and sputum specimens from humans with asthma [2]. However, human data on the relationship between ADMA and asthma are limited. Therefore, we examined the relationship between systemic ADMA levels and current asthma in a cohort from the Childhood Asthma Prevention Study (CAPS).

Study subjects included 314 8-year-old children from Sydney, Australia. They were originally enrolled prenatally into a randomised controlled trial of house dust mite avoidance and dietary fatty acid modification implemented from birth to 5 years. All subjects had one or more parents or an older sibling with asthma or wheezing illness. The details of this trial have been published elsewhere [3]. Of the 616 subjects who were enrolled prenatally, 314 had clinical and laboratory 


\section{TABLE 1 Baseline characteristics and L-arginine metabolic parameters in study subjects}

\begin{tabular}{|c|c|c|c|}
\hline & No current asthma & Current asthma & p-value \\
\hline Subjects $\mathrm{n}$ & 242 & 72 & \\
\hline FEV $1 \%$ pred & $105.4 \pm 11.1$ & $104.9 \pm 10.9$ & 0.75 \\
\hline FVC \% pred & $99.2 \pm 10.4$ & $99.7 \pm 11.1$ & 0.75 \\
\hline $\mathrm{FEV}_{1 / \mathrm{FVC}}$ & $0.93 \pm 0.05$ & $0.92 \pm 0.06$ & 0.089 \\
\hline Inhaled steroid use \% & 0.8 & 20.8 & $p<0.0001$ \\
\hline Atopy \% & 38.2 & 68.1 & $p<0.0001$ \\
\hline \multicolumn{4}{|l|}{ L-Arginine metabolism } \\
\hline ADMA $\mu \mathrm{mol} \cdot \mathrm{L}^{-1}$ & $0.91 \pm 0.23$ & $0.92 \pm 0.20$ & 0.88 \\
\hline $\mathrm{L}$-Arginine $\mu \mathrm{mo} / \mathrm{L}^{-1}$ & $106.1 \pm 31.5$ & $104.5 \pm 30.6$ & 0.70 \\
\hline L-Arginine/ADMA & $127.4 \pm 68.0$ & $122.2 \pm 59.7$ & 0.68 \\
\hline
\end{tabular}

data available for analysis at age 8 years for the present study. The study was approved by the local human research ethics committee and the parent or legal guardian gave written informed consent.
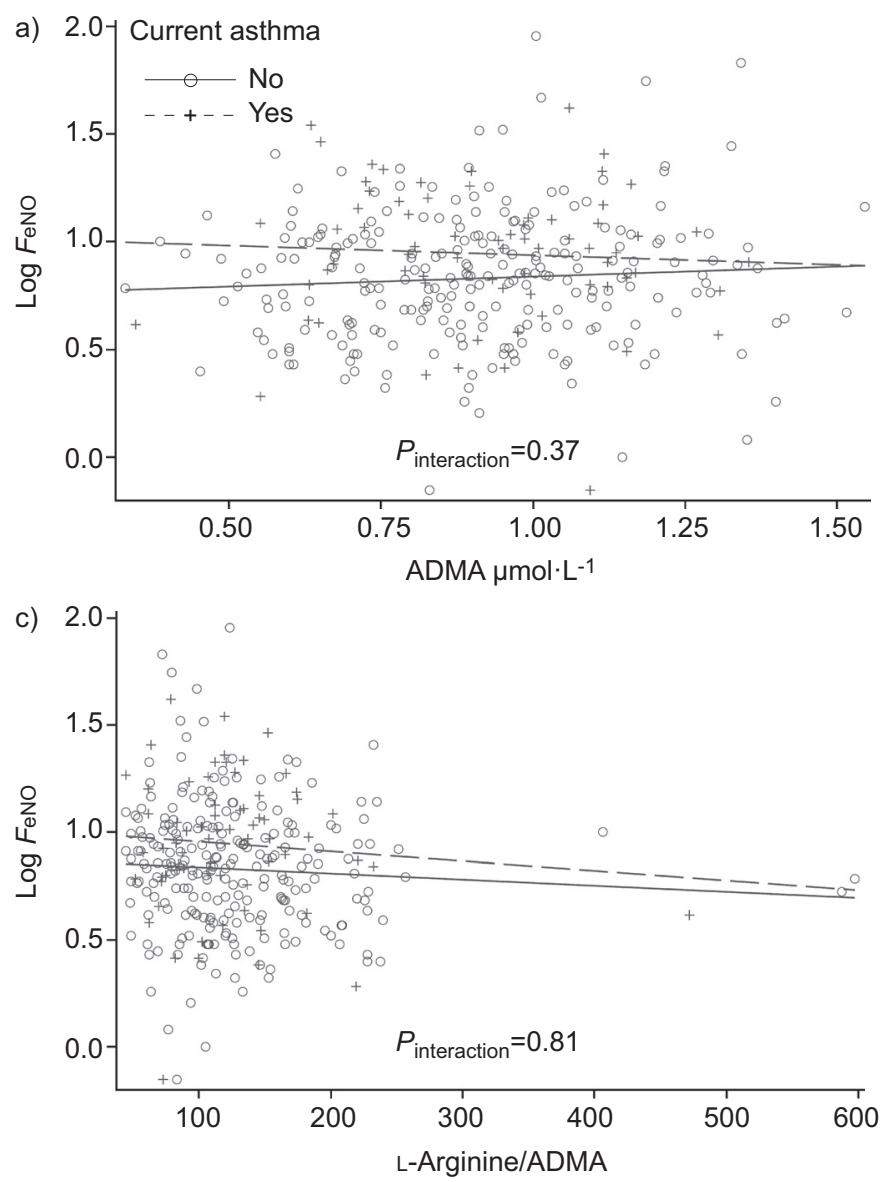

Current asthma at 8 years of age was defined as the presence of wheezing in the previous 12 months together with a reported doctor's diagnosis of asthma or the presence of airway hyperresponsiveness. Spirometry, airway hyperresponsiveness

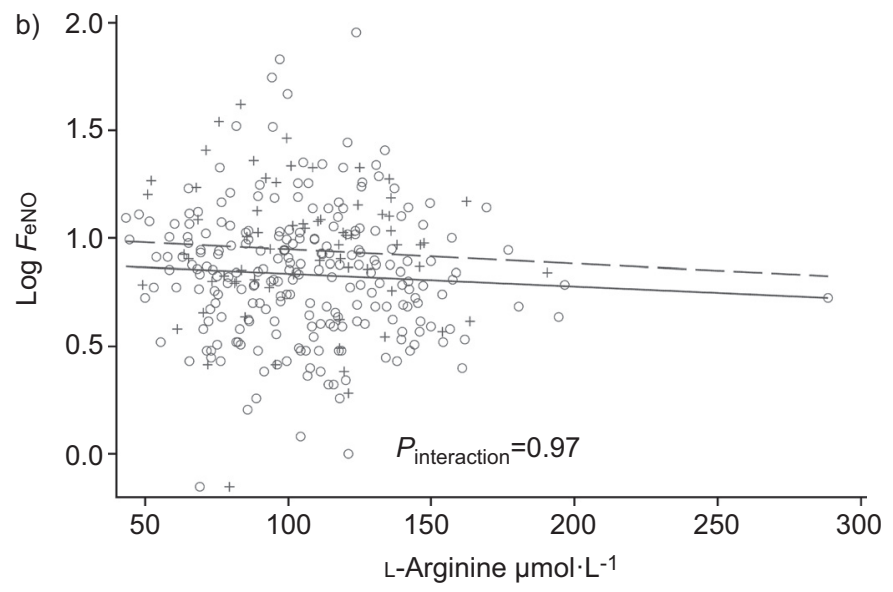

FIGURE 1. Association of fraction of expired nitric oxide ( $\mathrm{FeNO}$ ) with L-arginine metabolic parameters in 8-year-old children. No significant associations were found between $\log \left(F_{e N O}\right)$ with a) asymmetric dimethylarginine (ADMA) $\left.(p=0.42), b\right) L-$ arginine $(p=0.27)$ and $c)$ L-arginine/ADMA ( $p=0.21)$ for the entire study population. This lack of association was not influenced by the presence of current asthma ( $p$ value for interaction all $>0.37$ ). 
to methacholine, fraction of exhaled nitric oxide ( $F$ eNO) and atopic status were assessed, as described previously [4]. Serum was stored at $-80^{\circ} \mathrm{C}$ and analysed for ADMA by ELISA (Immunodiagnostik AG, Bensheim, Germany) and for L-arginine by high-performance liquid chromatography. Both ADMA and L-arginine were measured in duplicate and the averages were recorded.

Independent sample t-test (two-tailed) was used for comparisons between people with and without current asthma. Baseline variables that demonstrated skewing were logarithmically transformed prior to parametric testing. The associations between parameters of L-arginine metabolism and FeNO were analysed using general linear models, and the interaction with current asthma was examined. Statistical significance was determined by $\mathrm{p}$-value of $<0.05$.

Of the 314 children, 160 (51\%) were males and 72 (23\%) fulfilled our criteria for current asthma. Post-bronchodilator forced expiratory volume in $1 \mathrm{~s}$ (FEV1), forced vital capacity (FVC) and FEV1/FVC ratio were not significantly different between subjects with and without current asthma. However, children with asthma had higher FeNO concentrations, higher dose response ratio to methacholine (more airway responsiveness), a higher prevalence of atopy and were more likely to be taking inhaled corticosteroids than children without asthma (table 1).

ADMA and L-arginine levels were not significantly different between children with and without asthma. As ADMA competes with L-arginine for NOS utilisation, the L-arginine to ADMA ratio may be a better reflection of L-arginine bioavailability for nitric oxide production [5]. L-Arginine to ADMA ratio was also not significantly different between children with and without asthma (table 1).

$\log (\mathrm{FeNO})$ was not significantly associated with ADMA ( $\beta=0.06,95 \%$ CI $-0.09-0.2 ; p=0.42)$, L-arginine $(\beta=-0.001,95 \%$ CI -0.002-0.0005; $\mathrm{p}=0.27$ ) and $\mathrm{L}$-arginine to ADMA ratio $(\beta=-0.0003,95 \%$ CI $-0.0009-0.0002 ; p=0.21)$. No significant interaction with current asthma was present in these relationships (fig. 1).

Of those with current asthma, $21 \%$ had received daily inhaled corticosteroids therapy in the past 12 months. Inhaled corticosteroid use did not modify the results of any of the associations examined ( $p$-value for interaction all $>0.17$ ).

This study does not support the view that circulating levels of ADMA or L-arginine are related to the presence of asthma or airway inflammation in children. To the best of our knowledge, this is the largest study to examine ADMA and its relationship to asthma in children. The key strength of the study is its relatively large size in a well characterised group of 8-year-old children, thus enabling robust conclusions to be made regarding our results.

Recent studies have demonstrated a potential contribution of imbalances in L-arginine metabolism to the development of airway hyperresponsiveness and airflow obstruction in asthma [6-8]. ADMA is an endogenous inhibitor of all isoforms of NOS and is produced by the methylation of L-arginine residues by protein methyltransferases. The understanding of its possible role in asthma pathogenesis is evolving and has been supported predominantly by animal models of allergen-induced asthma.
SCOTT et al. [2], using a murine model of allergic airway disease, found upregulation of ADMA levels following ovalbumin challenge. Furthermore, exogenous administration of ADMA to challenge-naïve mice exaggerated their airway hyperresponsiveness to methacholine challenge. In another murine model, WELLS and co-workers $[7,8]$ showed that elevated circulating ADMA levels increased lung collagen deposition and exacerbated airway inflammation.

The results of our study appear to be in contrast to the currently available human data. A recent study from the Severe Asthma Research Programme found higher ADMA levels in adults with severe asthma, and measures of systemic L-arginine bioavailability correlated with the degree of airflow obstruction [6].This effect was confined to those with severe asthma, but not observed in those with mild-to-moderate asthma. MORRIS et al. [9], in a mixed paediatric and adult population, demonstrated a striking reduction in systemic L-arginine levels in asthmatics (less than $50 \%$ of control values) during acute exacerbations. More recently, Riccioni et al. [10] also found lower blood Larginine and ADMA levels in a paediatric asthma clinic population compared to controls. The discrepancy between our results and prior studies may be explained by the following. First, our study was a population-based cohort and, overall, those children with asthma in our study had a mild phenotype as evidenced by their well-preserved lung function. Secondly, although all children with current asthma had wheezing in the previous 12 months (by definition), they were not assessed during an acute exacerbation, and it is conceivable that systemic L-arginine metabolism may alter during disease exacerbations. Finally, the role of ADMA in asthma pathogenesis may also differ between children and adults.

We were only able to evaluate systemic ADMA and L-arginine levels as respiratory specimens were not collected in our study subjects. One important limitation of our study is that we cannot exclude the possibility that dysregulation of ADMA/ L-arginine metabolism may be compartmentalised to the airways and thus, no systemic spillover effects were detected in blood samples. Nevertheless, we believe that our study provides an important contrast to previous smaller clinic-based studies as alterations in systemic L-arginine metabolism were not evident in our population-based study of children with mild asthma. Finally, a recent small randomised controlled trial failed to demonstrate any beneficial clinical effects of oral L-arginine supplementation in moderate-to-severe asthma [11].

In conclusion, current asthma and airway inflammation were not associated with alterations in systemic ADMA and L-arginine levels in a large cross-sectional study of 8-year-old children. However, further studies involving examination of airway specimens are needed to clarify the role of ADMA in asthma pathogenesis.

\section{Edmund M.T. Lau*,\#, , Philip E. Morgan*, , Elena G. Belousova\#, Brett G. Toelle*, , Julian G. Ayer*, David S. Celermajer, and Guy B. Marks*,\#}

*Sydney Medical School, University of Sydney, Camperdown, ${ }^{\#}$ Woolcock Institute of Medical Research, Glebe, and "Heart Research Institute, Newtown, Australia. 
Correspondence: G.B. Marks, Woolcock Institute of Medical Research, PO Box M77, Missenden Road, Glebe, NSW 2050, Australia. E-mail: guy.marks@sydney.edu.au

Statement of Interest: None declared.

\section{REFERENCES}

1 Boger RH, Bode-Boger SM, Thiele W, et al. Biochemical evidence for impaired nitric oxide synthesis in patients with peripheral arterial occlusive disease. Circulation 1997; 95: 2068-2074.

2 Scott JA, North ML, Rafii M, et al. Asymmetric dimethylarginine is increased in asthma. Am J Respir Crit Care Med 2011; 184: 779-785.

3 Mihrshahi S, Peat JK, Marks GB, et al. Eighteen-month outcomes of house dust mite avoidance and dietary fatty acid modification in the childhood asthma prevention study (CAPS). J Allergy Clin Immunol 2003; 111: 162-168.

4 Toelle B, Ng KK, Crisafulli D, et al. Eight-year outcomes of the childhood asthma prevention study. J Allergy Clin Immunol 2010; 126: $388-389$.
5 Davis JS, Darcy CJ, Yeo TW, et al. Asymmetric dimethylarginine, endothelial nitric oxide availability and mortality in sepsis. PLoS One 2011; 6: e17260.

6 Lara A, Khatri SB, Wang Z, et al. Alterations of the arginine metabolome in asthma. Am J Respir Crit Care Med 2008; 178: 673-681.

7 Wells SM, Buford MC, Migliaccio CT, et al. Elevated asymmetric dimethylarginine alters lung function and induces collagen deposition in mice. Am J Respir Cell Mol Biol 2009; 40: 179-188.

8 Wells SM, Holian A. Asymmetric dimethylarginine induces oxidative and nitrosative stress in murine lung epithelial cells. Am J Respir Cell Mol Biol 2007; 36: 520-528.

9 Morris CR, Poljakovic M, Lavrisha L, et al. Decreased arginine bioavailability and increased serum arginase activity in asthma. Am J Respir Crit Care Med 2004; 170: 148-153.

10 Riccioni G, Bucciarelli V, Verini M, et al. ADMA, SDMA, L-arginine and nitric oxide in allergic pediatric bronchial asthma. J Biol Regul Homeost Agents 2012; 26: 561-566.

11 Kenyon NJ, Last M, Bratt JM, et al. L-Arginine supplementation and metabolism in asthma. Pharmaceuticals 2011; 4: 187-201.

DOI: $10.1183 / 09031936.00162212$ 